Electronic version of an article published as [International Journal of Production Economics, 2008, Vol. 113, No. 2, p. 957-968]

[DOI: http://dx.doi.org/10.1016/j.ijpe.2007.11.009] @ [copyright Elsevier] 


\title{
A MULTISTAGE SCENARIO OPTIMISATION PROCEDURE TO PLAN ANNUALISED WORKING HOURS UNDER DEMAND UNCERTAINTY*
}

\author{
Amaia Lusa ${ }^{\dagger 1}$, Albert Corominas ${ }^{1}$ and Norberto Muñoz ${ }^{2}$ \\ ${ }^{1}$ Institute of Industrial and Control Engineering (IOC), School of Industrial Engineering of Barcelona (ETSEIB), \\ Technical University of Catalonia (UPC), Barcelona, Spain. E-mail: amaia.lusa@upc.edu, \\ albert.corominas@upc.edu \\ ${ }^{2}$ Institute of Industrial and Control Engineering (IOC), Technical University of Catalonia (UPC), Barcelona, \\ Spain. E-mail: norberto.munoz@upc.edu
}

\begin{abstract}
The annualisation of working hours (i.e., the irregular distribution of the total number of working hours over the course of a year) makes it possible to adapt production capacity to fluctuations in demand. The required capacity, which is an essential data for the optimal planning of working time, usually depends on several complex factors. Often, it is impossible to reliably predict the required capacity or it is unrealistic to adjust it to a probability distribution. In some cases, it is possible to determine a set of required capacity scenarios, each with a related probability. This paper presents a multistage stochastic optimisation model that provides a robust solution (i.e., feasible for any possible scenario) and minimises the expected total capacity shortage.
\end{abstract}

Keywords: planning, uncertainty, optimisation, scenario analysis.

\footnotetext{
* ACKNOWLEDGMENTS:

Supported by the Spanish Ministry of Science and Technology (MCyT), project DPI2004-05797, co-financed by the ERDF.

The authors are grateful to Alberto García-Villoria for his help in performing the computational experiments. ${ }^{\dagger}$ CORRESPONDING AUTHOR:

Amaia Lusa, IOC, Av. Diagonal 647 (edif. ETSEIB), p.11, 08028 Barcelona, Spain. Tel. + 349340117 01; Fax.

+ 349340166 05; e-mail: amaia.lusa@upc.edu
} 


\section{Introduction}

The annualisation of working hours (AH) involves the irregular distribution of the total number of working hours over the course of a year. AH enables companies that operate in fluctuating markets to adapt capacity to demand and thus avoid expenses related to overtime, temporary workers or inventory. In services, $\mathrm{AH}$ prevents capacity from being wasted and workers from being fired during low-demand periods. In fact, according to a survey carried out by Oke (2000), most companies prefer AH over other flexibility schemes such as overtime, temporary workers or rejecting orders.

However, irregular working hours may cause working conditions to decline by making it harder for workers to plan their free time. Hence, an AH system must be the result of a bargaining process in which the company offers workers some kind of compensation (e.g., reduction in working time, salary increase, etc.) in exchange for the flexibility they provide. Furthermore, to avoid a significant decline in working conditions, laws and bargaining agreements often include bounds and rules that affect the distribution of working time. For example, the French law (see www.35h.travail.gouv.fr) states that the average number of working hours for any group over twelve consecutive weeks must not exceed $44 \mathrm{~h} /$ week.

To take full advantage of the flexibility allowed by $\mathrm{AH}$, the company must consider a sufficiently broad horizon (one year) when planning working time. Furthermore, it is very difficult to guarantee that the conditions are satisfied without carrying out the plan. Finally, working time planning is able to provide quantitative information (about savings, for example) that can be very useful when deciding to adopt an $\mathrm{AH}$ system, and when the company and the workers negotiate specific conditions.

AH gives rise to new problems that have hitherto been given little attention in the literature. For instance, Hung (1999a), Hung (1999b), Grabot and Letouzey (2000) and Azmat and Widmer (2004) pointed out that the concept of annualised hours is surprisingly absent from the literature on planning and scheduling. Due to the great variety of existing production systems, AH entails a considerable range of problems. Corominas et al. (2004a) discussed the characteristics of the planning problem and proposed a classification scheme, which gave rise to thousands of different cases. Moreover, $\mathrm{AH}$ often implies the need to solve a complicated working-time planning problem. Several authors have dealt with different versions of the 
problem (e.g., Hung, 1999a, Hung, 1999b, Gonçalves and Marçola, 2001, Corominas et al., 2002, Corominas et al., 2004b, Azmat and Widmer, 2004 and Azmat et al., 2004), but most papers (e.g., Lynch, 1995, MacMeeking, 1995 and Mazur, 1995) only discuss AH from a qualitative point of view.

To plan annualised working hours, it must be considered the demand for the products or services provided by a company. All of the procedures for planning working time proposed in the aforementioned papers are based on deterministic data sets or average values. However, demand (and hence the required capacity) usually depends on several complex factors. Furthermore, it is often impossible to reliably predict demand or it is unrealistic to adjust it to a probability distribution. In some cases, it is possible to determine a set of demand scenarios, each with its own related probability value. Failure to consider demand as a random variable may result in significant costs due to a capacity shortage or surplus when reality does not coincide with the forecast. Furthermore, by considering demand as a deterministic data, we may even obtain a solution that may be unfeasible depending on the real demand values.

To plan working time under demand uncertainty, stochastic programming (see Wets, 1989, Birge and Louveaux, 1997 and Ruszczynski and Shapiro, 2003)—in particular, scenariobased optimisation (see Rockafellar and Wets, 1987) —is an appropriate tool. The objective may be to optimise the expected value of a certain utility function (e.g., cost). It is also possible to include constraints that guarantee that the solution will be feasible for any possible scenario.

This paper presents a multistage scenario stochastic optimisation model that takes into consideration demand uncertainty when planning working time under annualised hours (AH). The rest of this paper is organised as follows: Section 2 introduces multistage scenario stochastic optimisation as a procedure for dealing with uncertain demand when planning working time under annualised hours; Section 3 describes the planning problem; Section 4 explains the stochastic optimisation model; Section 5 describes the computational study; finally, Section 6 presents the conclusions. 


\section{Multistage scenario stochastic optimisation}

Scenario-based optimisation has been used successfully in several cases to make decisions while taking into consideration possible future scenarios (see, for example, Ramos, 1992, Morton, 1996, Pallotino et al., 2002, Li et al., 2003, Mulvey et al., 2004 and Beraldi et al., 2006).

Most papers deal with two-stage programs (see the references listed at http://mally.eco.rug.nl/spbib.html for an updated survey on stochastic programming). However, multistage stochastic linear programs are the most studied and most frequently applied scenario-based stochastic models.

In the literature, there are few models for production-planning problems with demand uncertainty and, to the best of our knowledge, none of them consider working time as a variable to be planned (see Mula et al., 2006 for a review of production-planning models with uncertainty). For example, Eppen et al. (1989) model the strategic capacity-planning problem of a major automobile manufacturer using a stochastic programming approach based on demand scenarios; Escudero and Kamesam (1995) solved a scenario-based stochastic production-planning problem with demand uncertainty; Karabuk and Wu (1999) formulated a multistage stochastic program for a capacity-planning problem for a major semi-conductor manufacturer in which demand and capacity uncertainties were incorporated via a scenario structure.

When considering the problem of planning working time with annualised hours, the scenarios represent various possible capacity demands. In a multistage problem, at the beginning of each stage, a decision is made based on an uncertain future. By the end of the stage, some of the uncertain aspects for the future become apparent and the number of possible scenarios is reduced. Figure 1 represents this process. In the example (Figure 1), three stages are considered. At the beginning of the first stage (node $e_{0}$ ), we do not know which of the 12 possible scenarios will occur. At the beginning of the second stage (node $e_{1}, e_{2}$ or $e_{3}$ ), additional information is available (i.e., the actual demand for the periods corresponding to the first stage). This allows us to reduce the range of possible scenarios. For example, let us assume that, in scenarios 6 to 12, the demand is low for all periods corresponding to the first 
stage. If the demand has in fact been high, we can conclude that we are at node $e_{1}$ and we can discard scenarios 6 to 12. Figure 2 shows an example of the demand (or required capacity) for each scenario. Some scenarios have the same demand for a particular set of weeks. For example, if we reach node $e_{1}$, we cannot distinguish between scenarios 1 to 5 because the demand for these scenarios is the same as that for the weeks in the first stage (before reaching node $e_{1}$ ).

\section{[INSERT FIGURE 1]}

Figure 1. Scenario tree

\section{[INSERT FIGURE 2]}

Figure 2. Required-capacity scenarios

The planning model must indicate which decision must be made at each node. Thus, at the beginning, at node $e_{0}$, the model must provide all the decisions to be made at each of the rest of the nodes.

The number (or length) of stages may depend on several factors. When planning working time, the agreement between the company and the workers (collective bargaining agreement) must be taken into consideration.

For example, the agreement may establish a frozen period for the working hours so that the workers may plan their free time without being subjected to continuous changes in schedule. Thus, if the frozen period is one month, we cannot consider stages shorter than that because it would be impossible to maintain the working hours of a full month without changes. We do not know how many working hours will be assigned to the workers in a particular week of a stage until we know from which node we will start the stage.

There may also be a notice period. This means that workers must be notified in advance of the working hours they are assigned for a certain stage (e.g., from nodes $e_{2}, e_{3}$ or $e_{4}$ ). The notice period affects neither the scenario tree (Figure 1) nor the model presented in Section 4. 
Nonetheless, the information to be taken into account to plan a new stage (e.g., nodes $e_{2}, e_{3}$ or $e_{4}$ ) is actually available some time (notice period) before the beginning of that stage.

Figure 3 shows an example of the planning process. The length of the stages is equal to that of the frozen period. At time $t_{2}$, a new schedule for the weeks corresponding to Stage 2 and the following ones is determined and announced to the workers; at $t_{3}$, if new information is available, a new schedule for the weeks corresponding to Stage 3 and the following ones is determined and announced to the workers, and so forth.

\section{[INSERT FIGURE 3]}

Figure 3. Planning process

It is meaningless to consider nodes for which there is no additional information. In Figure 1, this is the case for node $e_{9}$. At node $e_{3}$, based on the available information, we know for certain that the real scenario is either 11 or 12 . At node $e_{9}$, it is still impossible to distinguish between scenarios 11 and 12. Thus, starting at node $e_{9}$, all the planning carried out for the periods in the last stage will be the same as that which can be carried out at the beginning of the second stage (at node $e_{3}$ ). This means that the number of stages between two consecutive nodes is not constant. In fact, the tree shown in Figure 1 could be considered a general graph in which the arcs do not represent periods of time but rather just connections between nodes. In this case, it is necessary to know which instant corresponds to each node.

\section{Problem statement}

Given the scenario tree (with the probabilities associated with each scenario and the required capacity for each scenario), the problem consists in determining the number of working hours for each worker, for each node and for each week corresponding to the stage beginning at that node.

The conditions that the solution must satisfy may come from a legal resolution or from a collective bargaining agreement between the management and the workers. As it is 
impossible to establish an exhaustive list of conditions a priori, we have considered the most common conditions in order to build a basic model for the problem. To adapt this model to a specific case, constraints can be added or deleted.

As an example, under the French law, the weekly number of working hours must fall within an interval defined by upper and lower bounds, the total number of annual working hours must be upper-bounded, and the average of the weekly working hours for any set of twelve consecutive weeks must be upper-bounded (this condition is assumed to apply to sets of twelve consecutive non-holiday weeks only).

The characteristics of the problem can be summarised as follows:

- Different workers may have different working hours.

- The number of annual working hours is upper-bounded (e.g., 1,750 hours).

- The number of weekly working hours is upper- and lower-bounded (e.g., [30-50] hours).

- The average number of working hours for any group of $L$ (e.g., twelve) consecutive working weeks may not exceed a certain value (e.g., 44 h/week).

- Workers take two holiday periods (two consecutive weeks in winter and four consecutive weeks in summer), which are arranged in advance.

- The expected value of the total capacity shortage must be minimised.

\section{Multistage scenario optimisation model}

We developed a linear program model based on the scenario-based optimisation scheme. The solution, which will be feasible for all scenarios, provides the decisions that must be made at each possible node and minimises the expected value of the total capacity shortage.

The notation used in this paper is defined as follows:

\section{Data:}

$T$ Number of periods (usually weeks) in the planning horizon (usually $\mathrm{T}=52$ weeks, which corresponds to one year). 
$E \quad$ Set of nodes $\left(e_{0}, e_{1}, \ldots, e_{0}\right.$ being the root node).

$\Gamma_{e}^{-} \quad$ Immediate precedent node of node $e\left(\forall e \in E \backslash e_{o}\right)$.

$\Gamma_{e}^{+} \quad$ Set of direct descendant nodes for node $e(\forall e \in E)$.

ED Set of nodes at which a decision must be made (i.e., all nodes but the last ones); $E D=\left\{e \in E \mid \Gamma_{e}^{+} \neq \varnothing\right\}$.

$S$ Set of scenarios, where each one is characterised by a path of the scenario tree (the root node, $e_{0}$, is not included). A path is a totally ordered set of nodes. In the example shown in Figure 1, the twelve scenarios are: $S=\left\{\left\{e_{1}, e_{4}, e_{10}\right\},\left\{e_{1}, e_{4}, e_{11}\right\},\left\{e_{1}, e_{5}, e_{12}\right\}\right.$, $\left\{e_{1}, e_{6}, e_{13}\right\},\left\{e_{1}, e_{6}, e_{14}\right\},\left\{e_{2}, e_{7}, e_{15}\right\},\left\{e_{2}, e_{7}, e_{16}\right\},\left\{e_{2}, e_{8}, e_{17}\right\},\left\{e_{2}, e_{8}, e_{18}\right\},\left\{e_{2}-e_{8}-e_{19}\right\}$, $\left.\left\{e_{3}, e_{20}\right\},\left\{e_{3}, e_{21}\right\}\right\}$. As explained above, node $e_{9}$ has been deleted.

$q_{s} \quad$ Probability that the scenario $s$ will occur $(\forall s \in S)$, with $\sum_{\forall s \in S} q_{s}=1$.

$T_{e}^{f}, T_{e}^{l}$ First and last period, respectively, corresponding to the stage beginning at node $e(\forall e$ $\in E D)$. Obviously, $T_{e}^{l}=T_{k}^{f}-1, \forall k \in \Gamma_{e}^{+}$

$p_{e} \quad$ Probability of reaching node $e\left(\forall e \in E \backslash e_{o}\right) ; p_{e}=\sum_{\forall s \in S \mid e \in S} q_{S}$.

$P \quad$ Set of paths, where each path is characterized by a totally ordered set of nodes at which a decision must be made. In the example shown in Figure 1 (but deleting node $\left.e_{9}\right)$, the six elements of the set are: $P=\left\{\left\{e_{0}, e_{1}, e_{4}\right\},\left\{e_{0}, e_{1}, e_{5}\right\},\left\{e_{0}, e_{1}, e_{6}\right\},\left\{e_{0}, e_{2}, e_{7}\right\}\right.$, $\left.\left\{e_{0}, e_{2}, e_{8}\right\},\left\{e_{0}, e_{3}\right\}\right\}$.

$C_{\text {tek }}$ Required capacity (in working hours), in period $t$, for the scenarios that include nodes $e$ and $k\left(\forall e \in E D ; \forall k \in \Gamma_{e}^{+} ; \forall t \in\left[T_{e}^{f}, \ldots, T_{e}^{l}\right]\right)$.

W Set of workers.

$A_{i} \quad$ Set of working (available) periods for worker $i, \forall i \in W$.

$H_{i} \quad$ Upper bound for the annual number of working hours for worker $i, \forall i \in W$.

$h m_{i t}$ Lower bound for the number of working hours for worker $i$ in period $t$ $\left(\forall i \in W ; \forall t \in A_{i}\right)$.

$h M_{i t}$ Upper bound $\left(>h m_{i t}\right)$ for the number of working hours for worker $i$ in period $t$ $\left(\forall i \in W ; \forall t \in A_{i}\right)$.

$L, h_{L}$ For each worker, the average number of working hours in a set of $L$ consecutive working periods must not exceed $h_{L}$ (with $L=12$ and $h_{L}=44$ hours). 
$L_{i} \quad$ For worker $i$, set of periods at which a group of $L$ consecutive working periods finishes $(\forall i \in W)$; i.e.: $j \in[L, \ldots, T] \mid t \in A_{i}, \forall t \in[j-L+1, \ldots, j]$

\section{Variables:}

$d_{\text {tek }} \quad$ Capacity shortage (in working hours) for period $t$, for the scenarios that include nodes $e$ and $k\left(\forall e \in E D ; \forall k \in \Gamma_{e}^{+} ; \forall t \in\left[T_{e}^{f}, \ldots, T_{e}^{l}\right]\right)$.

$x_{i t e} \quad$ Number of working hours for worker $i$ in period $t$ for the scenarios that include node $e$ $\left(\forall i \in W ; \forall e \in E D ; \forall t \in\left[T_{e}^{f}, \ldots, T_{e}^{l}\right] \cap A_{i}\right)$.

To sum up, when a node is reached, it must be determined how many working hours are to be done each period from that node to the end of the stage corresponding to that node. This is represented by the variables $x_{i t e}$. Once this decision has been made, shortages in future periods will depend on the actual required capacity (i.e., it will vary depending on which node is actually reached). These are represented by the variables $d_{t e k}$.

\section{Model:}

$$
\begin{aligned}
& {[M I N] z=\sum_{\forall e \in E D} \sum_{\forall k \in \Gamma_{e}^{+}} p_{k} \cdot \sum_{t=T_{e}^{f}}^{T_{e}^{l}} d_{t e k}} \\
& \sum_{\forall e \in S} \sum_{t \in\left[T_{e}^{f}, \ldots, T_{e}^{l}\right] \cap A_{i}} x_{i t e} \leq H_{i} \quad \forall i \in W ; \forall s \in P \\
& \left(\sum_{\forall i \in W \mid t \in A_{i}} x_{i t e}\right)+d_{t e k} \geq C_{t e k} \quad \forall e \in E D ; \forall k \in \Gamma_{e}^{+} ; \forall t \in\left[T_{e}^{f}, \ldots, T_{e}^{l}\right]
\end{aligned}
$$

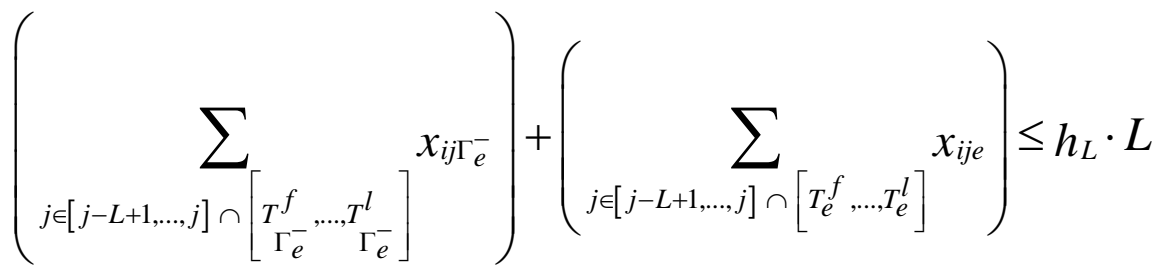

$$
\begin{aligned}
& \forall i \in W ; \forall e \in E D ; \forall t \in\left[T_{e}^{f}, \ldots, T_{e}^{l}\right] \cap L_{i} \\
& h m_{i t} \leq x_{i t e} \leq h M_{i t} \quad \forall i \in W ; \forall e \in E D ; \forall t \in\left[T_{e}^{f}, \ldots, T_{e}^{l}\right] \cap A_{i}
\end{aligned}
$$


The objective function to minimise (1) corresponds to the expected value of the total capacity shortage. Equation (2) imposes the upper bound on the annual number of working hours for each worker. This equation guarantees that the solution will be feasible for all possible scenarios. For each node $e$ and period $t$ belonging to the stage that begins at this node, equation (3) means that the planned capacity at this node plus the capacity shortage corresponding to period $t$ if descendant node $k$ is reached must be greater than or equal to the required capacity that the company would have at period $t$ if the actual scenario included node $k$. Equation (4) imposes the upper bound on the average number of working hours for each worker and set of $L$ consecutive working periods (note that includes the possibility that a block of $L$ periods is distributed over two consecutive stages). If short stages were used, this equation could easily be modified to include the possibility that the set of $L$ periods comprises the necessary number of stages. Lastly, (5) sets the upper and lower bounds on the number of working hours for each worker and period.

\section{Computational experiments}

We performed a computational experiment to evaluate the effectiveness of the stochastic model. The experiment had two main objectives: (1) to find out if the model can be solved in short times for instances of a realistic size (i.e., involving a large number of scenarios and nodes); and (2) to quantify the benefit of applying a scenario-based optimisation model rather than considering the required capacity as a deterministic value. For the second purpose we solved, for each instance, a deterministic model that took as a required capacity the expected value (considering the set of scenarios and their probabilities). The comparison criterion was the expected value of the total capacity shortage. For the stochastic model, this value is equal to the value of the objective function, while for the deterministic model it has to be computed from the obtained solution and the set of required capacity scenarios and their probabilities. The benefit of applying the stochastic model instead of the deterministic one (i.e., the value of the stochastic solution or VSS) can be then quantified by computing the difference between the expected value of the total capacity shortage obtained by solving the deterministic model and the one corresponding to the solution of the stochastic model, divided by the first one and multiplied by 100 .

A total of 360 instances were generated by combining the data (described below). 
- Planning horizon (T): 52 weeks (one year).

- Number of workers $(|W|): 10,50,100$.

- For each path and from each node, a required capacity is generated for each week belonging to the stage that starts at that node. The required capacity is set at random between $20 \cdot|W|$ and $50 \cdot|W|$.

- Number and length of stages: since the planning horizon is fixed, the number of stages depends on their length. As mentioned above, the length of the stages in a scenario tree is not constant. From a given node, the next stage may have a length of $l, 2 \cdot l, 3 \cdot l$ or $4 \cdot l$ with probabilities of $0.8,0.1,0.05$ and 0.05 . We considered two values for the basic stage length—l: 6 and 12 weeks.

- Three types of scenario tree were generated as follows (Figure 4 shows a scenario tree of each type):

- Type 1 (regular diversification): the number of nodes reachable from any given node is 2 or 3 , at random.

- Type 2 (diversification at the beginning): the number of nodes reachable from the nodes belonging to the first three stages is set at random between 3 and 6. For the following stages, the number of descendant nodes is 1 or 2 with probabilities of 0.75 and 0.25 , respectively. By generating the number of nodes in this manner, we obtain a tree that expands sharply at the beginning but less so much towards the end.

- Type 3 (diversification at the end): the number of nodes reachable from the nodes belonging to the first stages is set at 1 or 2 with probabilities of 0.75 and 0.25 , respectively. For the last three stages, the number of descendant nodes is set at random between 3 and 6. By generating the number of nodes in this manner, we obtain a tree that expands sharply at the end. 


\section{[INSERT FIGURE 4]}

Figure 4. Types of scenario tree

By generating the scenario trees in this manner, we obtain some instances with a very large number of nodes and scenarios. These instances may look unrealistic, but they are appropriate for testing the effectiveness of the optimisation model.

The experiment was performed using ILOG CPLEX 8.1 (using the dual simplex as a linear optimization method) and a Pentium IV at $3.4 \mathrm{GHz}$ with $512 \mathrm{MB}$ of RAM. The maximum computing time was set to 3,600 seconds. Of the 360 instances, 42 were too large to be solved (the computer ran out of memory). Figure 5 shows the number of workers, scenarios and nodes for the solved instances. The minimum, the average and the maximum number of variables was equal to 528, 39408 and 309077, respectively. For the constraints, these values were equal to 724, 63753 and 500572. Figure 6 shows the number of variables and constraints (i.e., size) for the solved instances.

\section{[INSERT FIGURE 5]}

Figure 5. Number of workers, scenarios and nodes for each instance

\section{[INSERT FIGURE 6]}

Figure 6. Number of variables and constraints for each instance

Figures 7 and 8 show the main results of the computational experiments. Figure 7 shows the computing times for the scenario-based model by number of workers, scenarios, nodes, variables and constraints. The number of scenarios, nodes, variables and constraints has a clearer influence on the computing time than does the number of workers. Overall, the proposed model can be solved in relatively short times for very large instances (probably larger than an actual instance would be).

The values of the stochastic solutions (VSS), in \%, for each instance, are plotted in Figure 8. The minimum, the average and the maximum values were equal to $0.001 \%, 17.45 \%$ and $100 \%$, respectively. As expected, using scenario analysis to consider uncertainty significantly reduces the expected value of the capacity shortage and the corresponding costs. 


\section{[INSERT FIGURE 7]}

Figure 7. Computing times (in seconds) by number of nodes, scenarios, workers, variables and constraints

\section{[INSERT FIGURE 8]}

Figure 8. Dotplot of savings (VSS, in \%)

We solved a small example to demonstrate the performance of the stochastic model. Its main characteristics are given below:

- Planning horizon: 36 weeks.

- Scenario tree (4 scenarios and 6 nodes): see Figure 9.

- Probabilities of each scenario (let qsi be the probability of scenario $i$ ):

- $\quad$ case 1 (qs1=qs2>qs3=qs4): qs1=qs2=0.35; qs3=0.15; qs4=0.15

- case 2 (qs1=qs2<qs3<qs4): qs1=qs2=0.15; qs3=0.3; qs4=0.4

- Number of workers: 20, which is equal to the number of workers needed to cover the expected value of the required capacity +1 (the number of workers needed to cover the maximum value of required capacity would be 25).

\section{[INSERT FIGURE 9]}

Figure 9. Scenario tree for the example

Figures 10 and 11 show the results of the example. PCe0, PCe1 and PCe2 correspond to the planned capacity for the decisions (i.e., working hours for each week and each worker) made at $e_{0}, e_{1}$ and $e_{2}$, respectively. At node $e_{0}$, we know nothing about the real scenario, whereas at node $e_{1}$ we know that the real scenario is either $\mathrm{s} 1$ or s2, and at node $e_{2}$ we know that the real scenario is either s3 or s4. The horizontal lines represent the maximum and minimum achievable capacity taking into account the upper and lower bounds for the weekly number of working hours.

In case 1 (Figure 10), in which the probability of scenarios 1 and 2 is greater than that of scenarios 3 and 4, the capacity for the weeks belonging to the first stage is adapted to the required capacity of the most probable scenarios (s1 and s2). At the end of the first stage, should the required capacity be that of scenarios 1 and 2 (i.e., node $e_{1}$ is reached), the required 
capacity for the following weeks will be low and the decision will be to adapt to the larger capacity of those required for scenarios 1 and 2 (with the number of workers available, this is perfectly feasible). However, if node $e_{2}$ is reached, the model tries to plan for the maximum number of working hours, which is not enough to cover the required capacity of scenarios 3 or 4 (the annual number of working hours is upper bounded).

In case 2 (Figure 11), in which scenarios 3 and 4 have the largest probability, during the first stage the capacity is adapted to the required capacity of scenarios 3 and 4 . In this case, when state $e_{2}$ is reached, the remaining number of annual working hours allows the model to adapt the capacity to the required capacity of scenario 4, which is the most probable.

Finally, for case 2, Figure 12 shows the planned capacity (PC) that corresponds to the solution of the deterministic model, which considers the expected value of the required capacity (represented in the figure by Sexp). AH makes it possible to adapt the planned capacity perfectly to Sexp. However, the solution is inadequate, particularly in the second stage, either because there is a significant capacity shortage (for scenarios 3 and 4) or surplus (for scenarios 1 and 2). For this particular example, the percentage of savings obtained by using our stochastic model rather than the deterministic model (i.e., the VSS, in \%) is equal to $59.27 \%$.

\section{[INSERT FIGURE 10]}

Figure 10. Solution for an example with four scenarios. Case 1: qs1=qs2>qs3=qs4

\section{[INSERT FIGURE 11]}

Figure 11. Solution for an example with four scenarios. Case 2: qs1=qs2<qs3<qs4

\section{[INSERT FIGURE 12]}

Figure 12. Solution of the deterministic model for an example with four scenarios. Case 2: qs1=qs2<qs3<qs4 


\section{Conclusions}

The annualisation of working hours allows companies to adapt their capacity to the required capacity. Linear programming tools, which have been tested in previous studies, are appropriate and efficient for planning working time and optimising the use of production resources (e.g., minimising costs due to capacity shortages) whilst satisfying a set of conditions.

The required capacity, which is an essential piece of data for planning working time, usually depends on several complex factors. Often, it is impossible to reliably predict the required capacity or it is unrealistic to adjust it to a probability distribution. In some cases, however, it is possible to determine a set of required capacity scenarios, each with its own related probability value.

This paper proposes a scenario-based optimisation approach for dealing with uncertain demand and presents a linear programming model that gives a robust solution (i.e., feasible for any scenario) and optimises the expected value of the total capacity shortage. The results of a computational experiment demonstrate that the model can be efficiently solved to optimality even for very large instances.

Finally, the expected value of the total capacity shortage obtained with the scenario-based model was compared with that obtained using the expected value of the required capacity with a deterministic model (i.e., the values of the stochastic solutions or VSS, in \%, were computed). Using the proposed stochastic model gives an average VSS of $17.45 \%$. We therefore conclude that the proposed model is an appropriate and useful tool for dealing with demand uncertainty when planning working time with annualised hours. 


\section{References}

Azmat, C. ; Widmer, M. (2004). A case study of single shift planning and scheduling under annualized hours: A simple three step approach, European Journal of Operational Research, Vol. 153, pp. 148-175.

Azmat, C.; Hürlimann, T.; Widmer, M. (2004). Mixed integer programming to schedule a single-shift workforce under annualized hours, Annals of Operations Research, Vol. 128, pp. 199-215.

Beraldi, P.; Ghiani, G.; Guerriero, E.; Grieco, A. (2006). Scenario-based planning for lotsizing and scheduling with uncertain processing times. International Journal of Production Economics, Vol. 101, No. 1, pp. 140-149.

Birge , J.R.; Louveaux, F. (1997). Introduction to Stochastic Programming. Springer-Verlag, New York.

Corominas, A.; Lusa, A.; Pastor, R. (2002). Using MILP to plan annualised hours, Journal of the Operational Research Society, Vol. 53, pp. 1101-1108.

Corominas, A.; Lusa, A.; Pastor, R. (2004a). Characteristics and classification of annualised working hours planning problems, International Journal of Services Technology and Management, Vol. 5/6, pp. 435-447.

Corominas, A.; Lusa, A.; Pastor, R. (2004b). Planning Annualised Hours with a Finite Set of Weekly Working Hours and Joint Holidays. Annals of Operations Research, Vol. 128, No. 14, pp. 217-233.

Eppen, G.D.; Martin, R.K.; Schrage, L. (1989). A scenario approach to capacity planning, Operations Research, Vol. 37, pp. 517-527.

Escudero, L.F.; Kamesam, P.V. (1995). On solving stochastic production planning problems via scenario modelling. TOP, Vol. 3, pp. 69-95.

Gonçalves, E.V.; Marçola, J.A. (2001). Annualized hours as a capacity planning tool in make-to-order or assemble-to-order environment: an agricultural implements company case. Production Planning \& Control, Vol. 12, pp. 388-398.

Grabot, B., Letouzey, A., 2000. Short-term manpower management in manufacturing systems: new requirements and DSS prototyping, Computers in Industry, Vol. 3, pp. 11-29. 


\section{http://mally.eco.rug.nl/spbib.html}

Hung, R., 1999a. Scheduling a workforce under annualized hours, International Journal of Production Research, Vol. 37, pp. 2419-2427.

Hung, R., 1999b. A multiple-shift workforce scheduling model under annualized hours, Naval Research Logistics, Vol. 46, pp. 726-736.

Karabuk, S.; Wu, D. (1999). Coordinating strategic capacity planning in the semiconductor industry, Technical Report 99T-11, Department of IMSE, Lehigh University.

Li, S.; Lolou, R.; Rahman, A. (2003). Technological progress and technology acquisition: Strategic decision under uncertainty. Production and Operations Management, Vol. 12, No. 1, pp. 102-119.

Lynch P., 1995. Annual Hours: An idea whose time has come, Personnel Management November, pp. 46-50.

MacMeeking J., 1995. Why Tesco's new composite distribution needed annual hours, International Journal Retail Distribution Management, Vol. 23, pp. 36-38.

Mazur L., 1995. Coming: the annual workweeks, Across the Board, Vol. 32, pp. 42-45.

Morton, D. P. (1996). An enhanced decomposition algorithm for multistage stochastic hydroelectric scheduling. Annals of Operations Research, Vol. 64, No. 0, pp. 211-235.

Mula, J.; Poler, R.; García-Sabater, J.P.; Lario, F.C. (2006). Models for production planning under uncertainty: A review. International Journal of Production Economics, Vol. 103, pp. 271-285.

Mulvey, J.; Shetty, B. (2004). Financial planning via multi-stage stochastic optimization. Computers and Operations Research, Vol. 31, No. 1, pp. 1-20.

Oke, A. (2000). Linking human resource flexibility with manufacturing flexibility: enablers of labour capacity flexibility in manufacturing plants. Proceedings of the First World Conference on Production and Operations Management (POM), Sevilla, August 27September 1.

Pallotino, P; Sechi, G.M.; Zuddas, P. (2002). A DSS for water resources management under uncertainty. Proceedings of the International Environmental Modelling and Software Society, Vol. 2. 
Ramos, A. (1992). Integrated model for capacity planning for manufacturing systems. Technical Report IIT-92-022, Universidad Pontificia de Comillas, Madrid.

Rockafellar, R.T.; Wets, R.J.-B. (1987). The principle of scenario aggregation in optimization under uncertainty, Manuscript, University of Washington, May 1987.

Ruszczynski, A.; Shapiro, A. (2003). Stochastic Progamming Models. In A. Ruszczynski and A. Shapiro Eds., Handbooks in OR \& MS, 10: Stochastic Programming, Chapter 1.

Wets, R.J.-B. (1989). Stochastic Programming, in Nemhauser, G.L. et al. (Eds.), Handbooks in OR\&MS, Vol. 1. Elsevier. pp. 573-629.

www.35h.travail.gouv.fr/ Ministére de l’Emploi et de la Solidarité, France, 15/09/2006. 


\section{LIST OF FIGURES}

Figure 1. Scenario tree

Figure 2. Required-capacity scenarios

Figure 3. Planning process

Figure 4. Types of scenario tree

Figure 5. Number of workers, scenarios and nodes for each instance

Figure 6. Number of variables and constraints for each instance

Figure 7. Computing times (in seconds) by number of nodes, scenarios, workers, variables and constraints

Figure 8. Dotplot of savings (VSS, in \%)

Figure 9. Scenario tree for the example

Figure 10. Solution for an example with four scenarios. Case 1: qs1=qs2 $>\mathrm{qs} 3=\mathrm{qs} 4$

Figure 11. Solution for an example with four scenarios. Case 2: qs1=qs2<qs3<qs4

Figure 12. Solution of the deterministic model for an example with four scenarios. Case 2: $\mathrm{qs} 1=\mathrm{qs} 2<\mathrm{qs} 3<\mathrm{qs} 4$ 


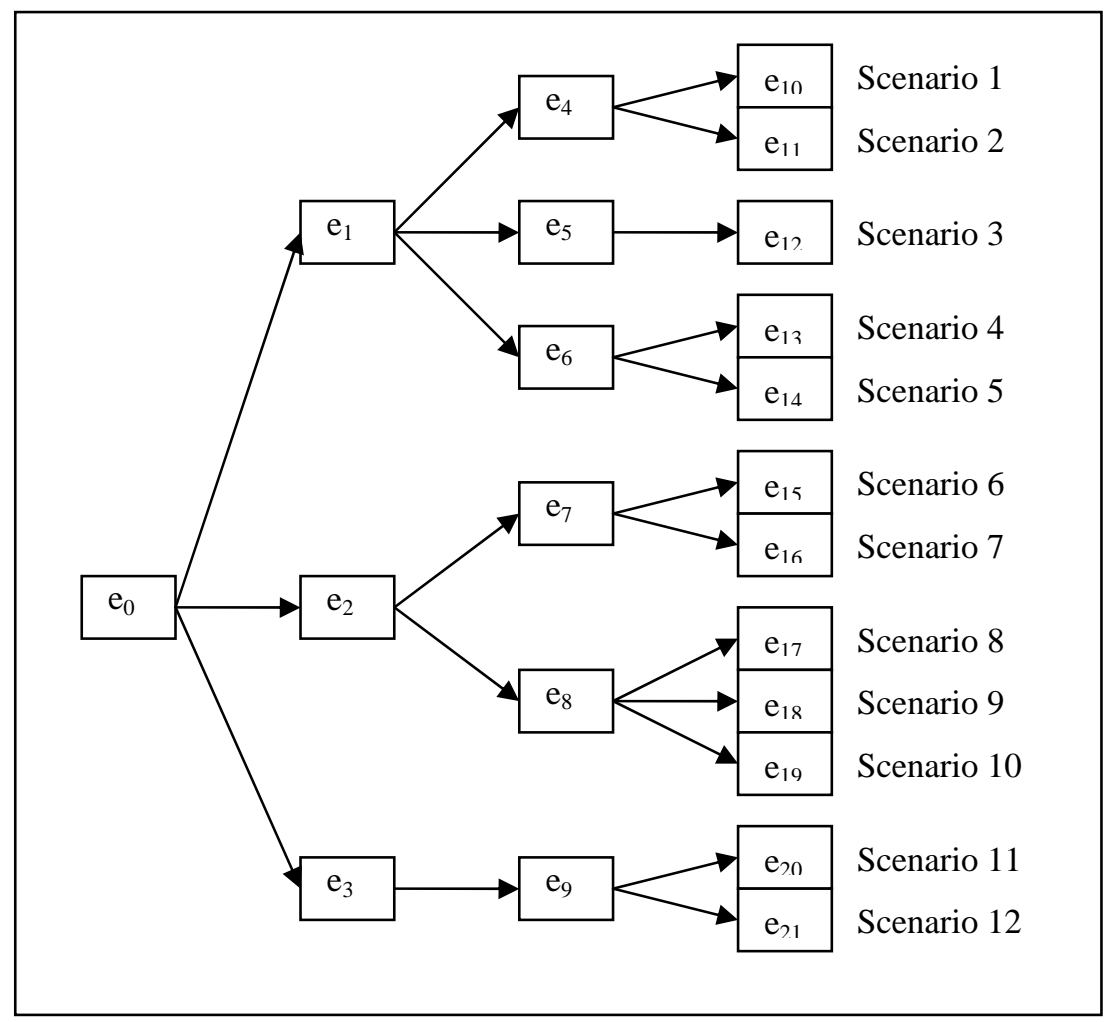

Figure 1. Scenario tree 
Figure 1. Scenario tree 


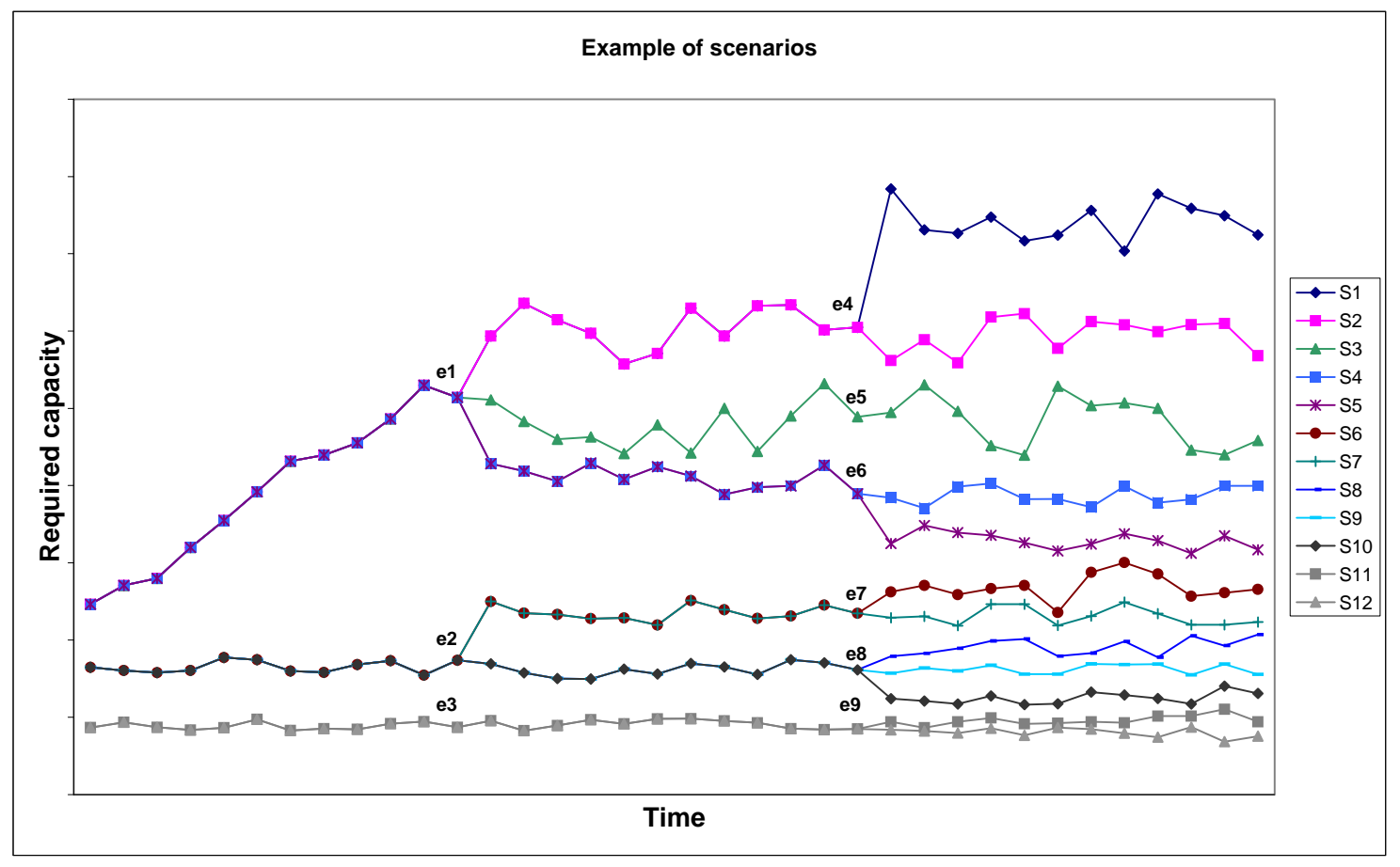

Figure 2. Required-capacity scenarios 
Figure 2. Required-capacity scenarios 


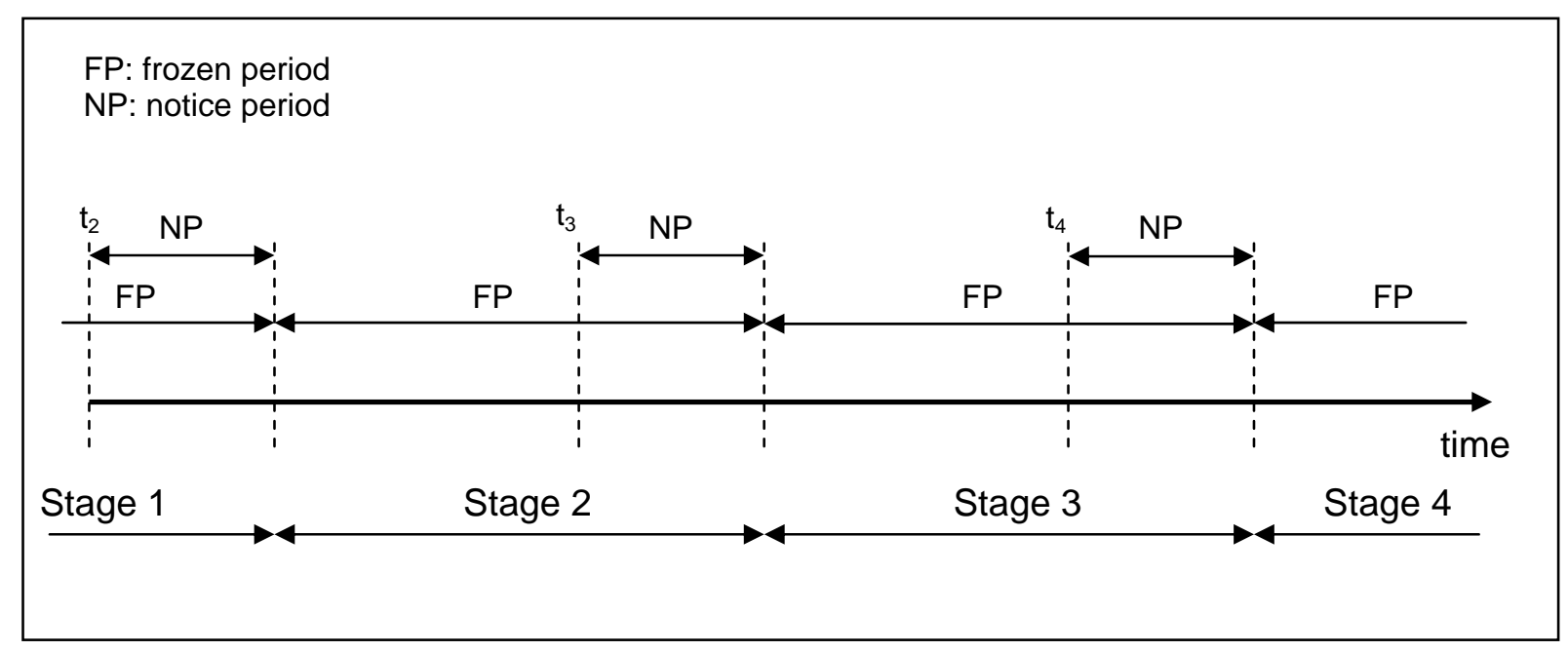

Figure 3. Planning process 
Figure 3. Planning process 


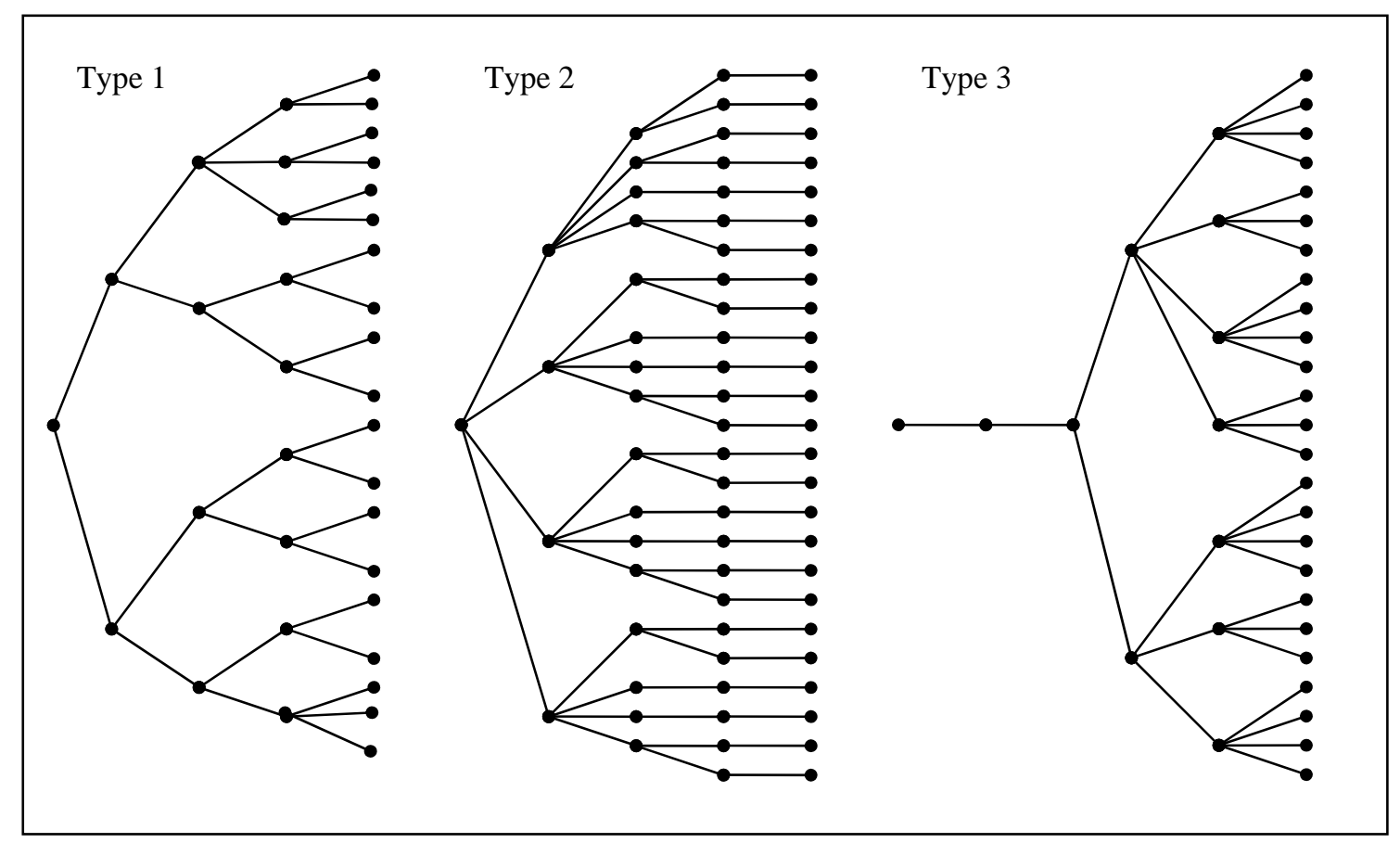

Figure 4. Types of scenario tree 
Figure 4. Types of scenario tree 


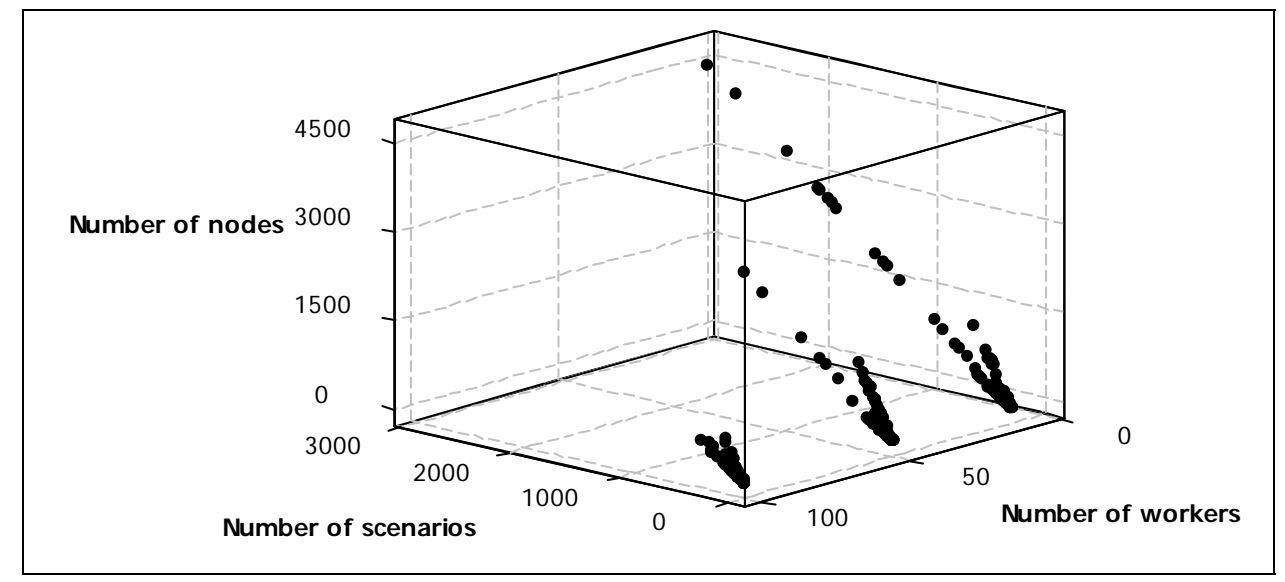

Figure 5. Number of workers, scenarios and nodes for each instance 
Figure 5. Number of workers, scenarios and nodes for each instance 


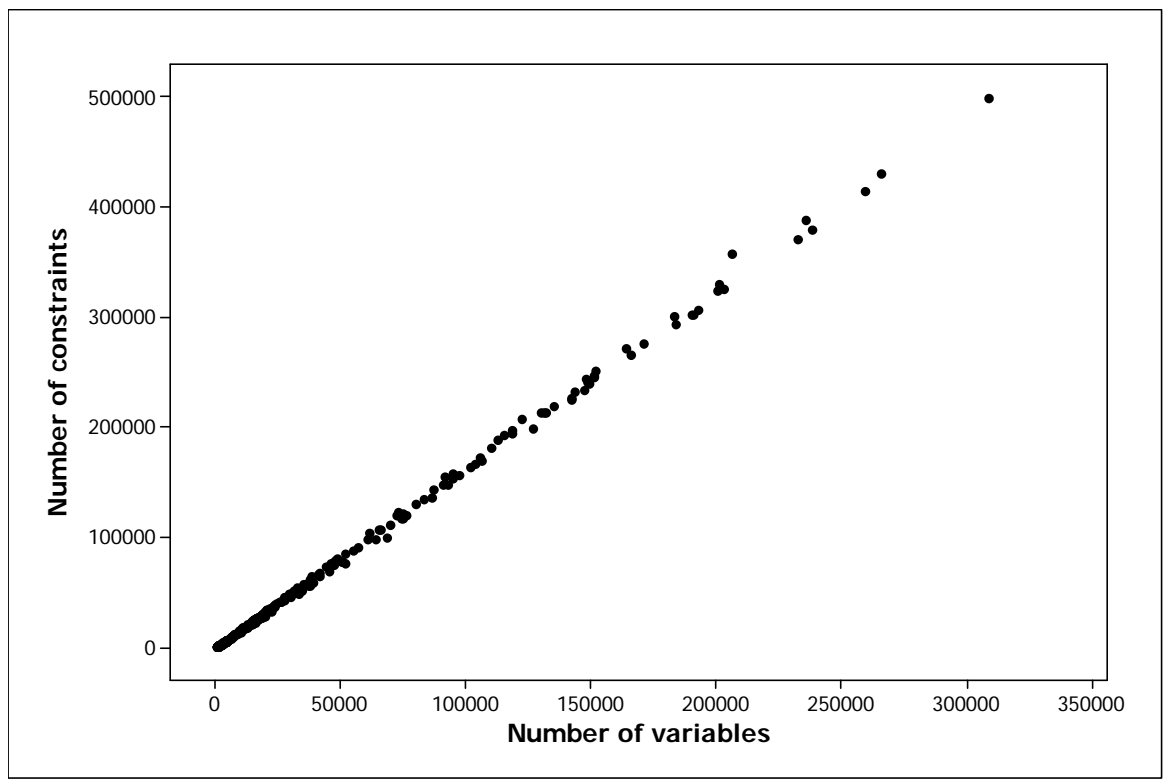

Figure 6. Number of variables and constraints for each instance 
Figure 6. Number of variables and constraints for each instance 


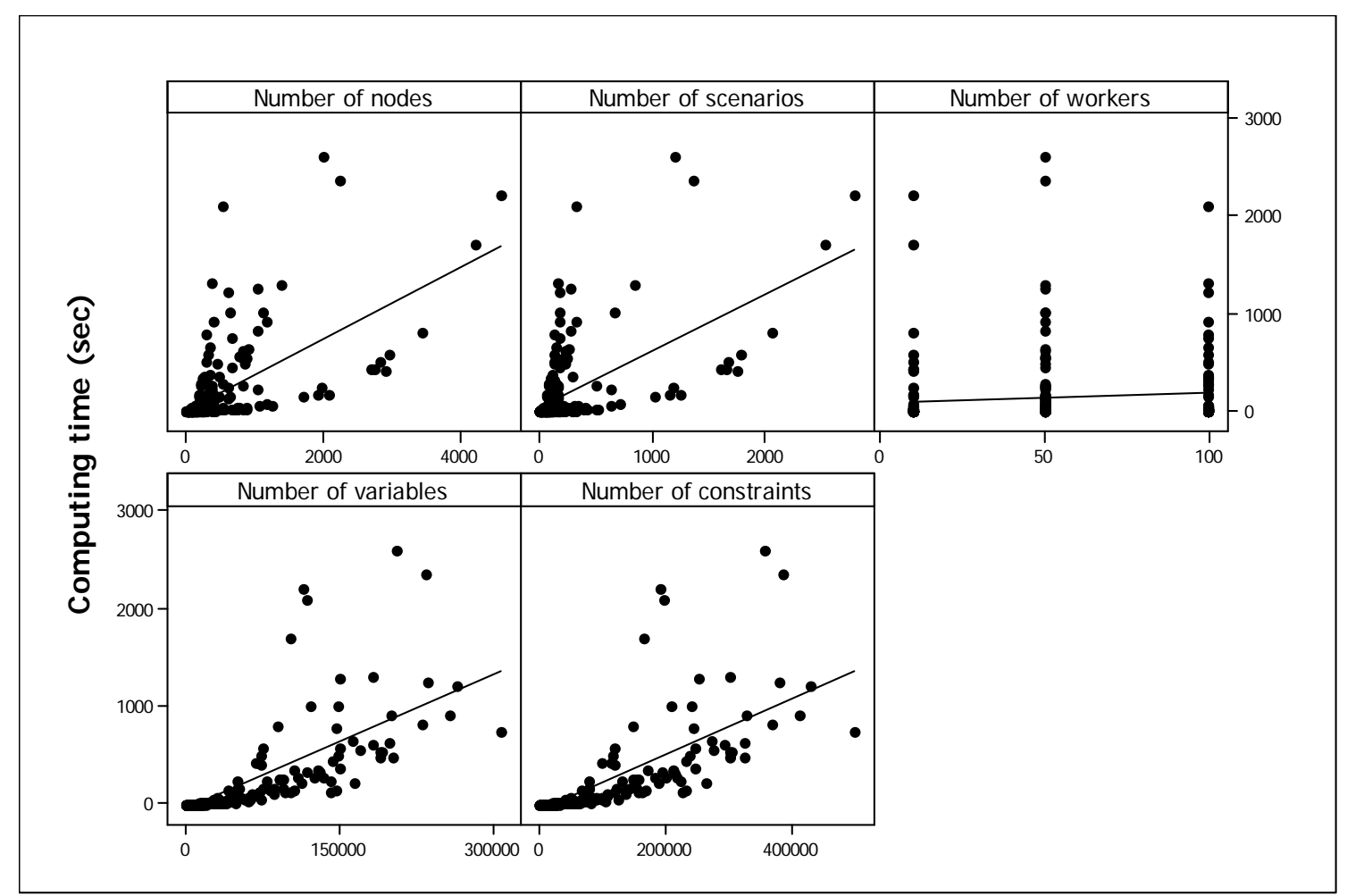

Figure 7. Computing times (in seconds) by number of nodes, scenarios, workers, variables and constraints 
Figure 7. Computing times (in seconds) by number of nodes, scenarios, workers, variables and constraints 


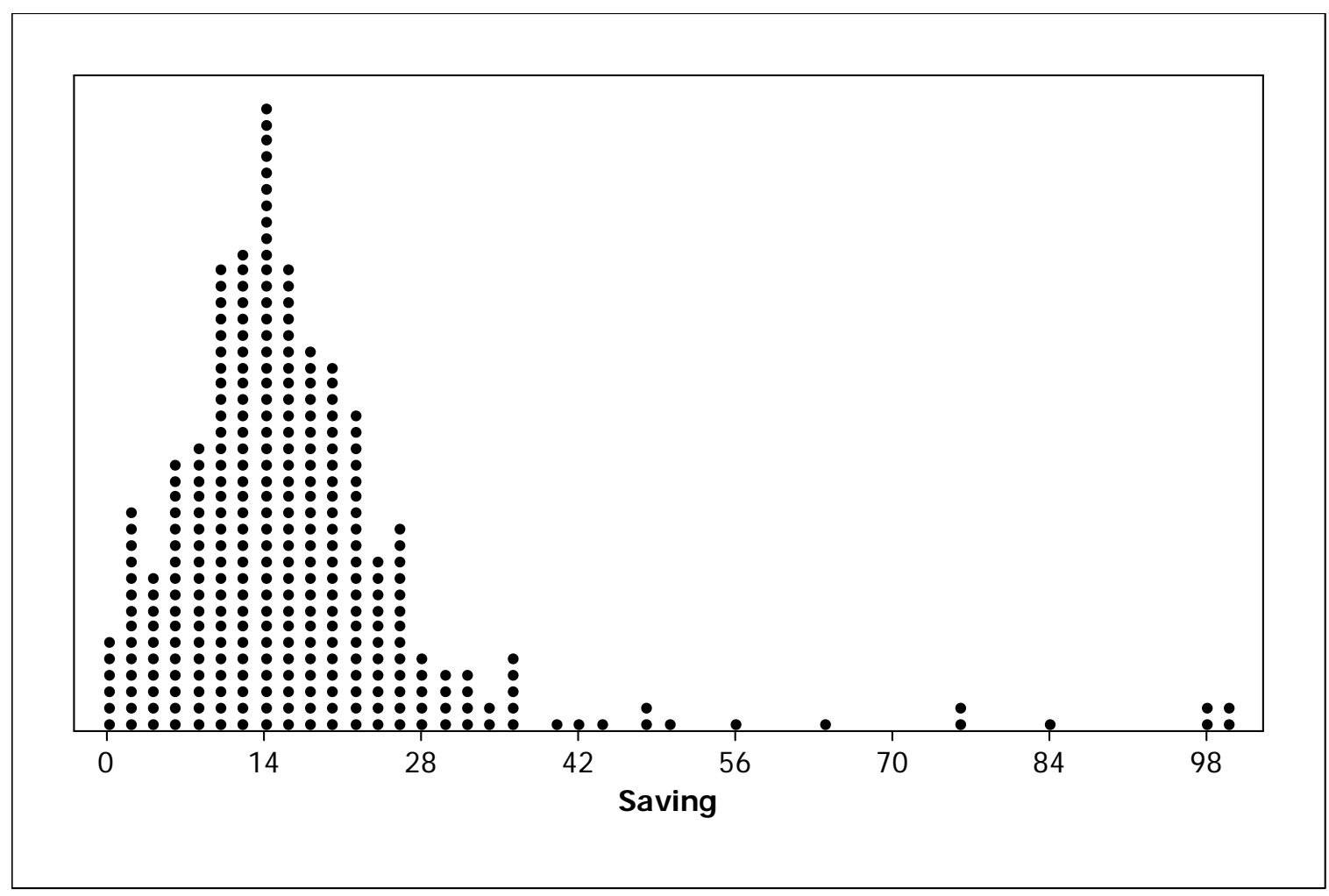

Figure 8. Dotplot of savings (VSS, in \%) 
Figure 8. Dotplot of savings (VSS, in \%) 


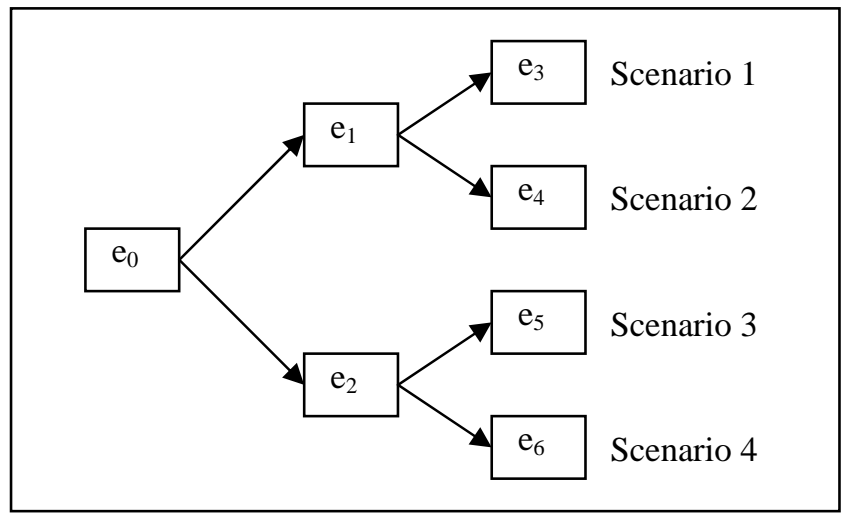

Figure 9. Scenario tree for the example 
Figure 9. Scenario tree for the example 


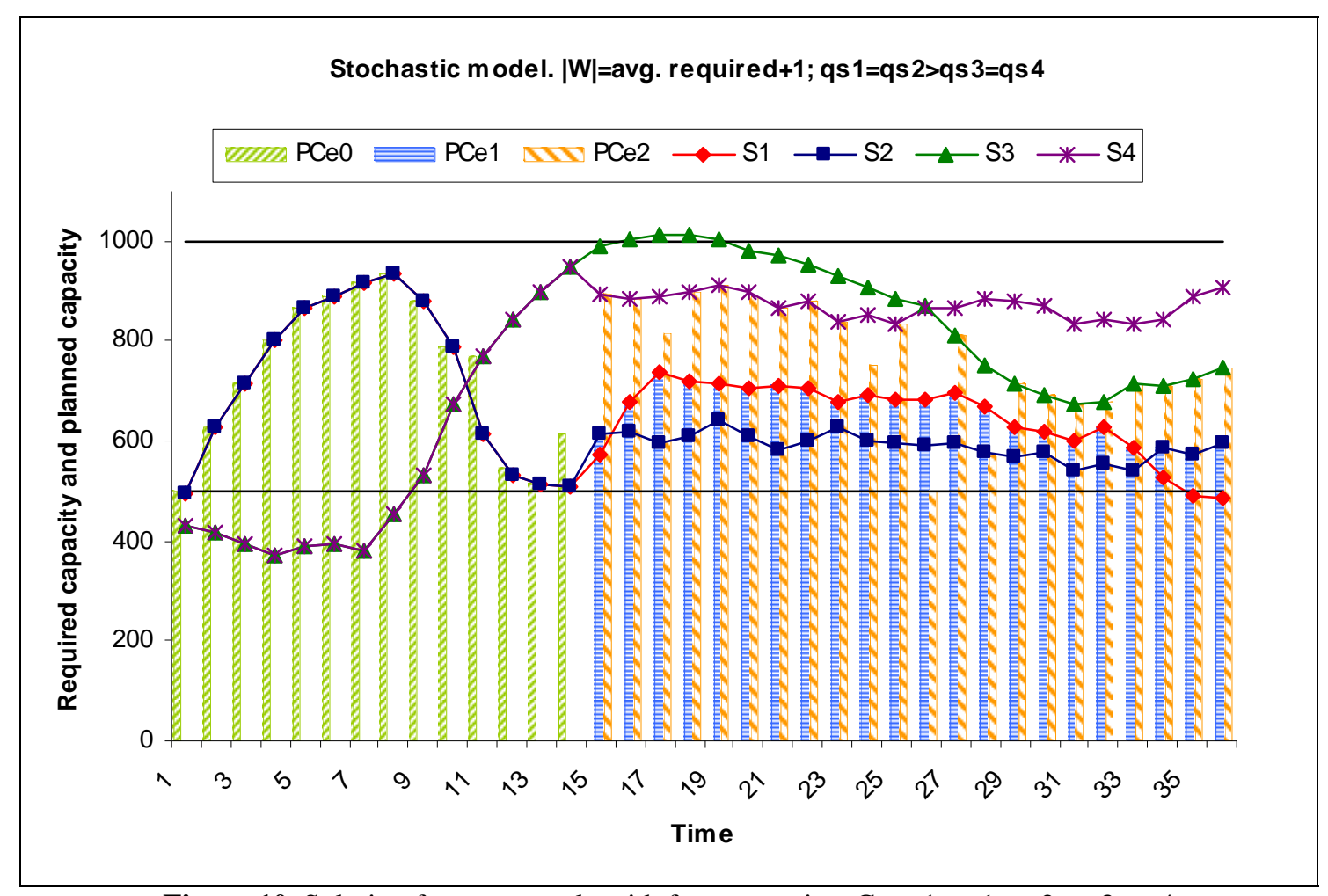

Figure 10. Solution for an example with four scenarios. Case 1: qs1=qs2>qs3=qs4 
Figure 10. Solution for an example with four scenarios. Case 1:

$$
\mathrm{qs} 1=\mathrm{qs} 2>\mathrm{qs} 3=\mathrm{qs} 4
$$






Figure 11. Solution for an example with four scenarios. Case 2: qs1=qs2<qs3<qs4 
Figure 11. Solution for an example with four scenarios. Case 2:

$$
\text { qs } 1=\text { qs } 2<\text { qs } 3<\text { qs } 4
$$




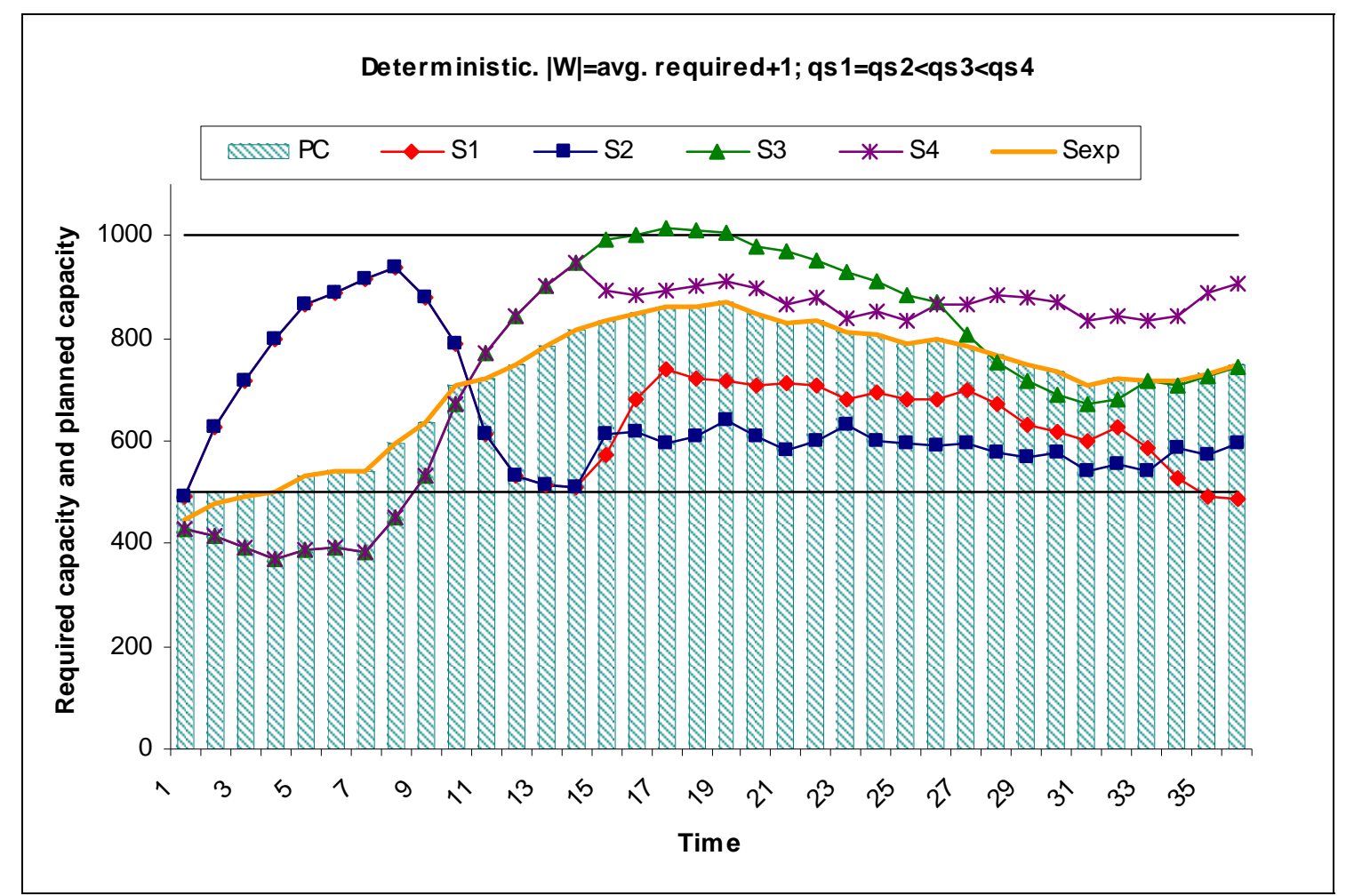

Figure 12. Solution of the deterministic model for an example with four scenarios. Case 2: qs1=qs2<qs3<qs4 
Figure 12. Solution of the deterministic model for an example with four scenarios. Case 2: qs1=qs2<qs3<qs4 